\title{
An Improved Airport Capacity Model Based on Historical Airport Acceptance Rate Analysis
}

\author{
Guozhuang Pan, Zhiyuan Shen ${ }^{+}$, Xinmiao Cheng and Zengyi Jiang \\ College of Civil Aviation, Nanjing University of Aeronautics and Astronautics, China
}

\begin{abstract}
As global economy grows, the number of planned flight largely increases at most airports. To improve the airport operation efficiency, the concept of airport acceptance rate (AAR) has been proposed. The conventional AAR researches only focus on evaluating the permitted arrival number of the airport. In this paper, the AAR and the traditional airport capacity model are firstly introduced. According to analyzing the historical acceptance rate data of US Memphis Airport, the airport operation mode is divided into 3 categories under different weather conditions. Based on different categories, an operation state decision factor is proposed to modify the airport capacity model. Finally, the revised model is used to calculate the excepted AAR of Chongqing Jiangbei Airport in China, which feature the same runway configuration as Memphis airport. Compared with the current airport capacity of Chongqing Jiangbei airport, some optimized suggestion are proposed.
\end{abstract}

Keywords: airport acceptance rate, airport capacity, weather condition, operation state decision factor.

\section{Introduction}

AAR is the arrival acceptance rate set by the traffic manager. If the AAR is set too low, it will lead to unnecessary delays. If it is set too high, it will make the controller to be overloaded. Airport Acceptance Rate (AAR) is a new concept proposed by ICAO (International Civil Aviation Organization) in 2012, document 9971. According to this document, AAR is a dynamic parameter that may varies from a wide range of factors. The airport acceptance rate is one of the current foreign airport capacity analysis models, and this kind of analysis model has no precedent in our country. Typically, AAR is determined by the previous weather conditions and the specific runway configuration, and is also set at the same time. AAR is set by people, so it can be seen as a person to determine the airport capacity. Therefore, the study of AAR can give us some views from the other perspectives of airport capacity.

In 1969, HARRIS established a capacity calculation model with random factors in it and considered the navigation device error and human factors in the model [1]. In 1970, R. S. Ratner for the first time expand the concept of runway capacity assessment to the terminal area and route traffic management and capacity assessment [2]. In 1998, David A. Lee, Caroline Nelson and Gerald Shapiro proposed the airport capacity and delay model based on the previous studies [3]. In 2008, DA Smith and DL Sherry used Support Vector Machines (SVM) to predict future airport capacity, compared the SVM to other classification methods and confirms that it is an effective way to predict airport capacity [4]. In 2010, Chung Stephanie and Daniel Murphy developed a model of airport acceptance rate to specialize airport acceptance rate [5]. In 2014, DeLaura, Richard and other people initially assess that wind energy can forecast airport acceptance rate and ground delay program (GDP) plan [6]. 2015, V. Ramanujam and H. Balakrishnan developed a statistical model that based on the discrete selection model framework and determined the various factors that influence the utility function of the decision maker [7]. In 2016, Yu Zhang proposed that under the IMC condition, the departure rate had a significant effect on AAR [8]. Airport capacity analysis model based on

Corresponding author. Tel.: +86-25-84893461; fax: +86-25-84893461.

E-mail address: shenzy@nuaa.edu.cn 
airport acceptance rate has become an effective tool for analyzing and improving airport capacity, and has been widely used in foreign airports.

Therefore, it is necessary to compare the domestic and foreign airports, combined with China's actual situation, then put forward the airport capacity promotion proposal and technical route to reduce the level of airport delays and enhance the airport rating.

\section{Airport Capacity Model}

\subsection{Single runway capacity model}

Single runway capacity model is described as follows [9]:

1) Instance when the approach speed of lead aircraft is higher than following aircraft $\left(V_{i}>V_{j}\right)$

Error free headway, $T_{i j}=T_{j}-T_{i}$, (no pilot and ATC controller error) assuming control is exercised as the lead aircraft passes the entry gate,

$$
T_{i j}=\frac{\delta_{i j}}{V_{j}}+\gamma\left(\frac{1}{V_{j}}-\frac{1}{V_{i}}\right)
$$

Position error buffer time (with pilot and ATC controller error) :

$$
B_{i j}=\sigma_{0} q_{v}-\delta_{i j}\left(\frac{1}{V_{j}}-\frac{1}{V_{i}}\right) \text { or } 0, \text { if } B_{i j}<0
$$

where $\delta_{\mathrm{ij}}$ is the minimum separation matrix; $\sigma_{0}$ is the standard deviation of the in-trail delivery error; $\mathrm{V}_{\mathrm{i}}$ is the speed of aircraft $i$ in knots; $V_{j}$ is the Following aircraft speed; $\gamma$ is the common approach length; $q_{v}$ is the value of the cumulative standard normal at probability of violation .

2) Instance when the approach of the lead aircraft is less than that of the Following aircraft $\left(\mathbf{V}_{\mathbf{i}}<\mathbf{V}_{\mathbf{j}}\right)$

Error free headway, $\mathrm{T}_{\mathrm{ij}}=\mathrm{T}_{\mathrm{j}}-\mathrm{T}_{\mathrm{i}}$ (no pilot and ATC controller error) with the minimum separation enforced when the lead aircraft passes the runway threshold,

$$
T_{i j}=\frac{\delta_{i j}}{V_{j}}
$$

Position error buffer time (with pilot and ATC controller error) is,

$$
B_{i j}=\sigma_{0} q_{v}
$$

Thus getting a mixed pattern of this two kinds of instance, where $\mathrm{ROT}_{\mathrm{i}}$ is the runway occupancy time for aircraft $\mathrm{i}(\mathrm{s})$. For $\mathrm{n}$ departures in $\mathrm{k}$ gaps, let $\varepsilon_{\mathrm{ij}}$ be the minimum departure-departure headway applied by air traffic control and let $\tau$ be the time delay (in seconds) for the departing aircraft. Adding the time delay term Equation becomes,

$$
\left(T_{i j}+B_{i j}\right)=\frac{\delta}{V_{j}}+R O T_{i}+(n-1) \varepsilon_{i j}+\tau .
$$

Since $\mathrm{T}_{\mathrm{ij}}+\mathrm{B}_{\mathrm{ij}}$ is calculated as an expected value in the analysis for arrivals only,

$$
E\left(\left(T_{i j}+B_{i j}\right) \geq E\left(\frac{\delta}{V_{j}}\right)+E\left(R O T_{i}\right)+(n-1) E\left(\varepsilon_{i j}\right)+E(\tau)\right.
$$

where $\delta$ is the Interval specification for takeoff / arrival aircraft.

\subsection{Narrow-distance double runway capacity model}

Narrow-distance double runway capacity model is described as follows:

1) Runway arrival capacity calculation of Runway NO.1 and NO.2.

As mentioned above, the length of the runway service time to the aircraft is affected by the gap of arrival and departure, so it is necessary to make an analysis of the time interval between taking off and arriving. The time interval for arriving aircraft is calculated as follows: 
First, the adjacent approach aircrafts (j-type aircraft Following i-type aircraft) are divided into the following two approach ways, respectively, to calculate their time interval:

(1)When $V_{i}>V_{j}$, we can get the interval between the two aircrafts by Eq.(1).

Taking the controller and the pilot's response time into account, it is necessary to add a buffer time $B_{i j}$, which is described by Eq. (2)

(2) When $V_{i} \leq V_{j}$, we can get the interval between the two aircrafts by Eq. (3) and (4).

After that, for $p_{i j}$, it is necessary to calculate the proportion of aircraft types - small aircraft, large aircraft, heavy aircraft in the airport. Set $\mathrm{a} \% 、 \mathrm{~b} \% 、 \mathrm{c} \%(\mathrm{a} \%+\mathrm{b} \%+\mathrm{c} \%=1)$. The models $\mathrm{i}, \mathrm{j}$, respectively, correspond the proportion of $\mathrm{I} \%, \mathrm{~J} \%$, so $p_{i j}=\mathrm{I} \% \times \mathrm{J} \%$.

Bring the value to the following Eq.,

$$
\mathrm{E}\left[T_{i j}+B_{i j}\right]=\sum_{i=1}^{n} \sum_{j=1}^{n} p_{i j}\left(T_{i j}+B_{i j}\right)
$$

where $p_{i j}$ is the ratio of two consecutive arriving aircraft whose lead is $\mathrm{i}$ and following is $\mathrm{j}$. Then we can get the weighted average of the landing time interval, so

$$
C_{\text {arrivals }}=\frac{1}{\mathrm{E}\left[T_{i j}+B_{i j}\right]} \text {. }
$$

2) Runway departure capacity calculation of NO.1 and NO.2.

When the time interval between the landing aircraft is long enough, the controller can insert the $n$-takeoff aircraft in accordance with the size $\mathrm{G}$ of the time gap. This interval (two adjacent landing aircraft) must meet Eq. (6). The interval provides with sufficient spacing to ensure that the air separation between arrival and departure aircraft does not violate the minimum clearance requirements. Since the narrow double runway make independent operation mode, there is no runway occupation time. So in this case, the Eq. can be modified as:

$$
\mathrm{E}\left[T_{i j}+B_{i j}\right] \geq E\left(\frac{\delta}{V_{j}}\right)+(n-1) E\left(\varepsilon_{i j}\right)+E(\tau) .
$$

When the j-type aircraft following i-type aircraft, the interval between the two aircrafts meet the above Eq., which can be inserted $n_{i-j}$ aircraft in it. Calculate the number of intervening aircraft that can be inserted between adjacent landing aircraft in each case. And then multiply the probability of j-type aircraft following the i-type aircraft and the number of slots within the hour unit can calculate the runway take-off aircraft capacity. The Eq. is as follows:

$$
C_{\text {departures }}=T G \sum_{i=1}^{n} \sum_{j=1}^{n} p_{i j}\left(n_{i j}\right)
$$

where $\varepsilon_{i j}$ is intervals between adjacent takeoff-aircraft; $\tau$ is time delay; $n_{i-j}$ is the number of takeoff-aircrafts that can be inserted between adjacent landing aircraft $\mathrm{i}, \mathrm{j} ; T G$ is the number of time slots per unit hour.

\section{Comparative analysis based on the airport acceptance rate}

For the Memphis Airport's saturated capacity: heavy (30\%) and small (70\%). Assume that the normal approach length is 7 miles. Radar surveillance is available with 20 seconds for the standard deviation of in-trail delivery accuracy error and a probability of violation of 5\%. And arrivals is random. Then considering the aircraft performance characteristics, we use the models to get the following results. Verified $\sum_{i, j} P_{i j}=1.0$. Therefore, the expected value of the headway is $E\left(T_{i j}\right)=139.7 \mathrm{~s}$. And then calculate the buffer time for successive approach. The expected value of the actual headways is $165.8 \mathrm{~s}$. The arrivals only capacity is

$$
C_{\text {arrivals }}=\frac{1}{E\left(T_{i j}+B_{i j}\right)} \text { vehicles per second }
$$

For the single runway example the arrivals-only capacity is $\mathrm{C}=3600 / 165.8=21.8$ aircraft arrivals per hour. Gaps can be studied for all four possible instances studied so far. For example, if a heavy aircraft is followed 
by a small one, there is a headway of 300 seconds between two successive arrivals. This leaves a large gap that be exploited by air traffic controllers to handle a few departures on the same runway. By Eq. (6), for $n$ departure between arrivals, when $\mathrm{n}$ is $1,2,3,4$, the minimum value of $E\left(T_{i j}+B_{i j}\right)$ is $120.8,198.8,276.8$ and $354.8(\mathrm{~s})$.We assume random arrivals. One departure (on the average) can be scheduled between a heavy aircraft followed by another heavy aircraft. Since 121 seconds are needed to schedule a departure (expected value for all types of operations), we conclude that one departure can be followed anytime by two heavy aircraft each other. Other cells are computed in a similar fashion. From our analysis of arrivals only, we determined that on the average hour 21.8 arrivals could be processed at the runway. Since two successive arrivals are needed to form a gap, we can infer that an average of 20.8 gaps are present in one hour. The probability of each one of the four arrival instances is known and has been calculated. Thus using these two pieces of information we estimate the number of times gaps will occur during one hour. Considering the instance of a heavy aircraft leading another heavy aircraft, nine percent of the time this instance occurs at the airport. Thus for 20.8 gaps per hour this represents an equivalent number of hourly departures per arrival instance $\left(E D_{H-H}\right)$,

$$
E D_{H-H}=T G\left(P_{H-H}\right)\left(D G_{H-H}\right)
$$

where TG is the total number of gaps per hour, $P_{H-H}$ is the probability that a heavy aircraft follows another heavy, and $D G_{H-H}$ is the number of departures per gap for each instance. By the data we get, the total departures is 25.2 departures per hour.These results indicate that a single runway can process 21.8 arrivals per hour and during the same period process 25.2 departures per hour using the gaps formed by the arrivals. Total operations $=47$ aircraft per hour.If only departures are processed at this runway (no arrivals), the departures only capacity is the reciprocal of the departure headway (78 seconds), $C_{\text {dep }-N A}=46.2 / \mathrm{h} \quad$.Airport engineers use a capacity diagram illustrated in the figure to display all three hourly capacity results in a single diagram. These diagrams represent a Pareto frontier of arrivals and departures. The airport can be operated inside the Pareto boundary.

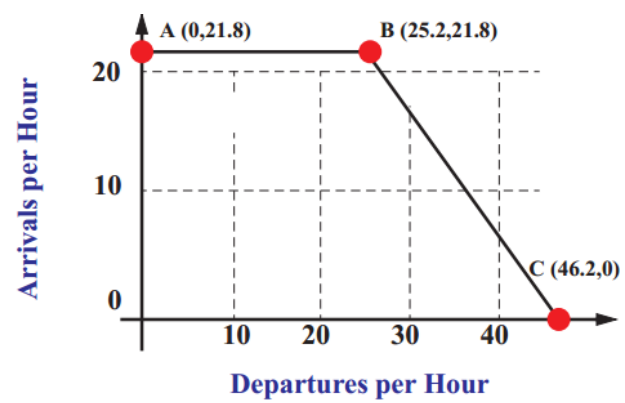

Fig. 1: All three runway hourly capacity.

For the two narrow-distance parallel runways in the three runways of Memphis International Airport, the capacity analysis was carried out using the parallel approach-independent departure. For each arrival on the secondary runway we need to account for possible buffers (or position errors) since controllers do not have a fast update of the aircraft position in their radar scopes. The aircraft landing in the secondary runway thus poses a higher challenge to the air traffic controller because they require two buffers computed between arrivals in the primary runway. The minimum expected gap without buffers allowing an aircraft arrival on the secondary runway is calculated to be 5,320 meters (using simple geometry). A 5,320 meters distance translates into the following headways for each one of the three aircraft groups operating at this facility:

Table 1: The facility the three aircraft groups operating at

\begin{tabular}{|c|c|c|c|}
\hline & Heavy & Large & Small \\
\hline $\mathrm{T}$ gap(second) & 9 & 74 & 103 \\
\hline
\end{tabular}

Add two buffers of 33 seconds to simulate probability of violations of 5\% (consistent with human factor studies). This brings the minimum gap for an arrival on the second runway to be 147 seconds. Now find gaps between successive arrivals on the primary runway with at least a gap of 140 seconds. Knowing the 
probability matrix for both runways, we can estimate the number of gaps where sufficient headway exit allowing and arrivals on the secondary runway. The approach is similar to which executed in the Excel program to estimate departures in the mixed mode case. If all conditions are met as stated, the airport can process 32 arrivals per hour. To estimate the number of departures when the arrivals is 9.2 per hour we turn our attention to the original Pareto diagram for the primary runway only. The figure suggests that if arrivals are processed at a rate of $9 / \mathrm{hr}$, we could process 33 departures/hr on the same runway. This provides a first estimate of the number of departures on the secondary runway when 9 arrivals are processed in the same runway. The primary runway handles 17 departures and 23 arrivals per hour. Therefore, the new close-parallel configuration will handle 50 departures and 32 arrivals on two runways. Historical statistics are based on the operational data of Memphis International Airport and Chongqing Jiangbei International Airport from April to September in 2015, and are counted throughout the day (24 hours). The purpose of historical data analysis is to analyze the historical operation of these two airports from an objective point of view. The results can be supported by the conclusion.

For Memphis International Airport, select 3: 00-4: 00 (the most departure) and 23: 00-24: 00 (the most approach) to do the main analysis. For Chongqing Jiangbei International Airport, select 7: 00-8: 00 (the most departure) and 15: 00-16: 00 (balance) to do the main analysis.

Corresponding to three different weather conditions (normal, weather reasons, and the weather is close to the marginal weather) in each period we get 3 different values of $\mu$ and set the parameters

$$
\mu=\frac{A A R_{\text {standard }}}{\overline{A A R}}
$$

where $\varphi=\frac{1}{\mu}$, we finally get the results as Tab. 2 .

Table 2: Compare result between memphis airports and Chongqing Jiangbei airport

\begin{tabular}{|c|c|c|c|c|c|c|c|c|c|c|c|c|}
\hline \multirow{3}{*}{$\begin{array}{c}\text { Parameters } \\
\text { Time Period } \\
\overline{A A R}\end{array}$} & \multicolumn{6}{|c|}{ Memphis International Airport } & \multicolumn{6}{|c|}{ Chongqing Jiangbei International Airport } \\
\hline & \multicolumn{3}{|c|}{ 3: $00-4: 00$} & \multicolumn{3}{|c|}{ 23: 00-24: 00} & \multicolumn{3}{|c|}{ 7: 00-8: 00} & \multicolumn{3}{|c|}{ 15: $00-16: 00$} \\
\hline & 40.55 & 39 & 35 & 30.32 & 28 & 25 & 31.76 & 30 & 25 & 19.49 & 18 & 15 \\
\hline$A A R_{\text {standard }}$ & \multicolumn{3}{|c|}{50} & \multicolumn{3}{|c|}{32} & \multicolumn{3}{|c|}{40} & \multicolumn{3}{|c|}{25.06} \\
\hline$\mu$ & 1.23 & 1.28 & 1.43 & 1.06 & 1.14 & 1.28 & 1.25 & 1.33 & 1.6 & 1.29 & 1.39 & 1.67 \\
\hline$\varphi$ & 0.81 & 0.78 & 0.7 & 0.95 & 0.88 & 0.78 & 0.79 & 0.75 & 0.63 & 0.77 & 0.72 & 0.6 \\
\hline
\end{tabular}

From the parameter value, the ratio of actual departure capacity and theoretical departure capacity of 7 : 00-8: 00 in Chongqing Jiangbei Airport are close to the 3: 00-4: 00 in Memphis Airport, Indicating that release times are enough or even close to saturation in the busy hours. Compared with the ratio of actual approach capacity and theoretical approach capacity of 15: 00-16: 00 in Chongqing Jiangbei Airport and 23: 00-24: 00 in Memphis Airport, we can see there is a lot of room for improvement. Despite the runway configuration and weather conditions are similar, Chongqing airport actual approach capacity and theoretical approach capacity are still different. It is necessary to improve the approach capacity in the actual implementation of the scheduling, such as paired approach, re-divide the flight sector and design new flights procedures, and increase simulator training for controllers to improve proficiency.

\section{Conclusion}

This paper first summarizes the current situation of airport capacity at home and abroad based on airport acceptance rate, studies airport acceptance rate, inspects and interprets FAA's concept of airport acceptance rate, and analyzes the factors that affect airport acceptance rate. Then establish the single runway and narrow double runway airport capacity analysis mode based on airport acceptance rate. The airport acceptance rate and airport capacity of Memphis Airport and Chongqing Jiangbei Airport were calculated, and compare these two airports theoretical capacity of the actual operation. And finally propose recommendations and development direction for the Chongqing airport to enhance the capacity. 


\section{Acknowledgements}

The authors would like to thank the financial support by National Natural Science Foundation of China (No.61501225).

\section{Reference}

[1] HARRIS R M. Models for runway capacity analysis[R].Washington DC :Mitre Corporation,1969,45-52.

[2] R.S. Ratner. A methodology for evaluating the capacity of air traffic control systems[R].FAA-RD-70-69,1970:1-175.

[3] David A Lee, Caroline Nelson, Gerald Shapin. The aviation system analysis capability airport capacity and delay models[R].NASR/CR,207659,1998:56-67.

[4] DA Smith and DL Sherry. Decision Support Tool for Predicting AircraftArrival Rates, Ground Delay Programs, and Airport Delays from Weather Forecasts, [R] .10.1109/ICNSURV.2008.

[5] S. Chung and D. Murphy, "Developing a model to determine called rates at airports," in AIAA Aviation Technology, Integration, and Operations (ATIO) Conference, [C] .2010.

[6] R.A. DeLaura, R.F. Ferris, F.M. Robasky, S.W. Troxel and N.K. Underhill. Initial Assessment of Wind Forecasts for Airport Acceptance Rate (AAR) and Ground Delay Program (GDP) Planning. Prepared for the Federal Aviation Administration, [C].Washington, D.C. 20591.

[7] Varun Ramanujam and Hamsa Balakrishnan. Data-Driven Modeling of the Airport Configuration Selection Process, [R] .10.1109/THMS.2015.

[8] Yu Zhang. Comparison of Airport Capacity and Analysis of Airport Acceptance Rate (AAR)-- Ongoing U.S. and China Collaborative Efforts,[Z].2016.

[9] Dr. Antonio A. Trani, Airport Capacity - Introduction, Civil and Environmental Engineering Virginia Polytechnic Institute and State University, Blacksburg, Virginia,2012. 